\title{
Some Probability Density Functions and Their Characteristic Functions
}

\author{
By Frank McNolty
}

\begin{abstract}
This paper presents, without derivation, several generalized density functions together with their characteristic functions. The densities are expressed variously in terms of special functions such as: $I_{\nu}(x)$, the modified Bessel function of the first kind of order $\nu$; $K_{\nu}(x)$, the modified Bessel function of the second kind of order $\nu ;{ }_{1} F_{1}(a ; b ; x)$, the confluent hypergeometric function; ${ }_{2} F_{1}(a, b ; c ; x)$, the hypergeometric function; $W_{a, b}(x)$, Whittaker's function; $\Phi_{3}(\beta ; \gamma ; b x, c x)$, a generalized hypergeometric function (type I);

$\Phi_{2}(b, c, d ; \gamma ; \lambda x, \tau x, \beta x)$,

a generalized hypergeometric function (type II); and $\phi_{\lambda}^{\mu}\left(b v^{\mu}\right)$, a generalized Bessel type function. The first five cases are summarized from the work of Laha [7], Pearson [25] and Raj [26] while Cases 13 through 19 have not previously appeared in the literature of statistics or Fourier transforms. In what follows, the usual notation $f(x)$, for a density function, and $\varphi(t)$, for a characteristic function, will be used with all parameters considered as real quantities:

$$
\varphi(t)=\int_{-\infty}^{\infty} \exp (i t x) f(x) d x
$$

Case 1. Laha [7], Bose [2], Bose [3], Erdélyi [4], McKay [14], McNolty [15]-[22]. In this case and in Cases 3, 6-12 and 15-18, the function $f(x)=0$ for $x<0$.

$$
\begin{gathered}
f(x ; \lambda, \gamma, Q)=2^{Q / 2-3 / 2} \frac{x^{(Q-1) / 2}}{\gamma^{0-1}} \lambda^{0} \cdot \exp \left(-\frac{\gamma^{2}}{4 \lambda}-\frac{\lambda}{2} x\right) \cdot I_{Q-1}\left[\gamma\left(\frac{x}{2}\right)^{1 / 2}\right], x \geqq 0, \\
\gamma \geqq 0, \lambda \geqq 0, Q>0, \\
\varphi(t)=\left(1-\frac{2 i t}{\lambda}\right)^{-Q} \cdot \exp \left[\frac{i t \gamma^{2}}{2 \lambda^{2}\left(1-\frac{2 i t}{\lambda}\right)}\right] .
\end{gathered}
$$
\end{abstract}

Case 2. Pearson [25], Bhattacharyya [1], Bose [3], Erdélyi [4], Sastry [6], Laha [7], McKay [14], McNolty [20].

$$
\begin{gathered}
f(x ; a, \nu)=\frac{a(|x| a)^{\nu} K_{\nu}(|x| a)}{2^{\nu} \pi^{1 / 2} \Gamma\left(\nu+\frac{1}{2}\right)}, \quad-\infty<x<\infty, \\
a>0, \nu>-\frac{1}{2}, \\
\varphi(t)=a^{2 \nu+1} /\left(t^{2}+a^{2}\right)^{\nu+1 / 2} .
\end{gathered}
$$

Case 3. Raj [26], McNolty [20].

Received July 21, 1972.

AMS (MOS) subject classifications (1970). Primary 65E05, 60G50, 44A20; Secondary 33A30, $33 \mathrm{~A} 40,33 \mathrm{~A} 70,42 \mathrm{~A} 36$. 


$$
\begin{gathered}
f(x ; a, b ; \lambda, \mu)=a^{\lambda+1} x^{\lambda} \cdot \exp \left(-a x-\frac{b}{a^{\mu}}\right) \cdot \phi_{\lambda}^{\mu}\left(b x^{\mu}\right), \quad x \geqq 0, \\
a>0, \mu \geqq-1, b \geqq 0, \lambda>0
\end{gathered}
$$

where

$$
\phi_{\lambda}^{\mu}\left(b x^{\mu}\right)=\sum_{r=0}^{\infty} \frac{\left(b x^{\mu}\right)^{r}}{r ! \Gamma(1+\lambda+\mu r)},
$$

$$
\varphi(t)=\left(1-\frac{i t}{a}\right)^{-\lambda-1} \cdot \exp \left\{-\frac{b}{a^{\mu}}\left[1-\left(1-\frac{i t}{a}\right)^{-\mu}\right]\right\} \text {. }
$$

Case 4. Laha [7].

$$
\begin{aligned}
& f(x ; a, b, c)=e^{-c^{2 / 2}} \frac{x^{(a+b) / 2-1}}{2^{(a+b) / 2}} \sum_{r=0}^{\infty} \frac{(-) c^{2 r} x^{r}}{r ! \Gamma(a+r) 2^{2 r}} W_{(a-b) / 2,(a+b+2 r-1) / 2}(2 x), \\
& =(-1) \cdot[\text { expression }(7 a) \text { with } x \text { replaced by }(-x) \\
& \text { and } \Gamma(a+r) \text { replaced by } \Gamma(b+r)], \quad x<0, \\
& \varphi(t)=(1-i t)^{-a} \cdot(1+i t)^{-b} \cdot \exp \left[-\frac{c^{2} t^{2}}{2\left(1+t^{2}\right)}\right] \text {, } \\
& a, b>0 ; c \geqq 0 ; a+b>1 \text {. }
\end{aligned}
$$

Here, expressions (7a) and (7b) differ from the result given in [7].

Case 5. Laha [7]. Letting $a=b$ in expression (7) gives

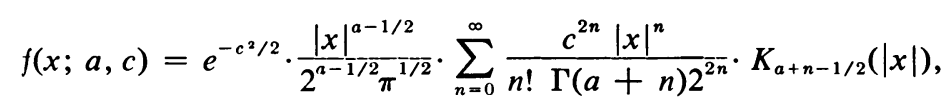

$$
-\infty<x<\infty
$$

$$
\begin{gathered}
a>0, c \geqq 0, \\
\varphi(t)=\left(1+t^{2}\right)^{-a} \cdot \exp \left[-c^{2} t^{2} / 2\left(1+t^{2}\right)\right] .
\end{gathered}
$$

Case 6. Erdélyi [4], McNolty [19]-[21].

$$
\begin{gathered}
f(x ; a, b ; \nu, \mu)=\frac{a^{\nu} b^{\mu}}{\Gamma(\nu+\mu)} e^{-b x} x^{\mu+\nu-1} \cdot{ }_{1} F_{1}[\nu ; \nu+\mu ;(b-a) x], \quad x \geqq 0, \\
\mu+\nu>0 ; a, b \geqq 0 ;\left\{\begin{array}{l}
b>a, \nu>0, \nu+\mu>0(\mu \text { may be negative }) ; \\
a>b, \mu>0, \nu+\mu>0(\nu \text { may be negative }) ;
\end{array}\right. \\
\varphi(t)=a^{\nu} b^{\mu} /(a-i t)^{\nu}(b-i t)^{\mu} .
\end{gathered}
$$

Case 7. Erdélyi [4], McNolty [20].

$$
\begin{gathered}
f(x ; a, b ; \nu)=\frac{\frac{\pi^{1 / 2}\left(b^{2}-a^{2}\right)^{\nu+1 / 2}}{2^{\nu} \Gamma\left(\nu+\frac{1}{2}\right) a^{\nu}} e^{-b x} x^{\nu} I_{\nu}(a x), \quad x \geqq 0,}{b>a>0, \nu>-\frac{1}{2},} \\
b>a+10
\end{gathered}
$$


where expression (13) is a special case of (11).

$$
\varphi(t)=\left(b^{2}-a^{2}\right)^{\nu+1 / 2} /\left[(b-i t)^{2}-a^{2}\right]^{\nu+1 / 2} .
$$

Case 8. Erdélyi [4], McNolty [20].

$$
\begin{gathered}
f(x ; a, b ; \nu)=\frac{\pi^{1 / 2}\left(b^{2}-a^{2}\right)^{\nu+3 / 2}}{2^{\nu+1} \Gamma\left(\nu+\frac{3}{2}\right) a^{\nu} b} e^{-b x} x^{\nu+1} I_{\nu}(a x), \quad x \geqq 0, \\
b>a>0, \nu>-1, \\
\varphi(t)=(b-i t)\left(b^{2}-a^{2}\right)^{\nu+3 / 2} / b\left[(b-i t)^{2}-a^{2}\right]^{\nu+3 / 2} .
\end{gathered}
$$

Case 9. Erdélyi [4], McNolty [20].

$$
\begin{gathered}
f(x ; a, b, c ; \beta, \gamma)=\frac{a^{\gamma-\beta}(a-b)^{\beta}}{\Gamma(\gamma)} x^{\gamma-1} \cdot \exp \left(-a x-\frac{c}{a}\right) \cdot \Phi_{3}(\beta ; \gamma ; b x, c x), \\
a>b \geqq 0, c \geqq 0, \gamma>0, \beta \geqq 0,
\end{gathered}
$$

where

$$
\begin{gathered}
\Phi_{3}(\beta ; \gamma ; b x, c x)=\sum_{m=0}^{\infty} \sum_{n=0}^{\infty} \frac{(\beta)_{m}}{(\gamma)_{m+n} m ! n !} b^{m} c^{n} x^{m+n} \\
\varphi(t)=\frac{(a-b)^{\beta}(a-i t)^{\beta-\gamma}}{a^{\beta-\gamma}(a-b-i t)^{\beta}} \cdot \exp \left[\frac{i t c}{a(a-i t)}\right] .
\end{gathered}
$$

Case 10. Erdélyi [4].

$$
\begin{aligned}
& f(x ; a, b, c, d ; \beta, \gamma, \lambda, \tau)=\frac{(a-\lambda)^{b}(a-\tau)^{c}(a-\beta)^{d}}{\Gamma(\gamma) a^{b+c+d-\gamma}} \cdot e^{-a x} x^{\gamma-1} \\
& \cdot \Phi_{2}(b, c, d ; \gamma ; \lambda x, \tau x, \beta x), \quad x \geqq 0, \\
& a>\lambda, a>\tau, a>\beta ; \lambda, \tau, \beta \geqq 0 ; a>0, \gamma>0 ; b, c, d \geqq 0,
\end{aligned}
$$

where

$$
\Phi_{2}(b, c, d ; \gamma ; \lambda x, \tau x, \beta x)=\sum_{m=0}^{\infty} \sum_{n=0}^{\infty} \sum_{r=0}^{\infty} \frac{(b)_{m}(c)_{n}(d)_{r}}{(\gamma)_{m+n+r} m ! n ! r !} \lambda^{m} \tau^{n} \beta^{r} x^{m+n+r} \text {, }
$$

$$
\varphi(t)=\frac{(a-\lambda)^{b}(a-\tau)^{c}(a-\beta)^{d}(a-i t)^{b+c+d-\gamma}}{(a-i t-\lambda)^{b}(a-i t-\tau)^{c}(a-i t-\beta)^{d} a^{b+c+d-\gamma}} .
$$

Case 11. Erdélyi [4], McNolty [20].

$$
\begin{aligned}
& f(x ; a, b, c ; \gamma ; \lambda, \tau)=\frac{(a-\lambda)^{b}(a-\tau)^{c}}{\Gamma(\gamma)^{b+c-\gamma}} \cdot e^{-a x} x^{\gamma-1} \cdot \Phi_{2}(b, c ; \gamma ; \lambda x, \tau x), \\
& x \geqq 0, \\
& a>\lambda, a>\tau ; a, \gamma>0 ; b, c, \lambda, \tau \geqq 0,
\end{aligned}
$$

where 


$$
\begin{gathered}
\Phi_{2}(b, c ; \gamma ; \lambda x, \tau x)=\sum_{m=0}^{\infty} \sum_{n=0}^{\infty} \frac{(b)_{m}(c)_{n}}{(\gamma)_{m+n} m ! n !} \lambda^{m} \tau^{n} x^{m+n}, \\
\varphi(t)=\frac{(a-\lambda)^{b}(a-\tau)^{c}(a-i t)^{b+c-\gamma}}{(a-i t-\lambda)^{b}(a-i t-\tau)^{c} a^{b+c-\gamma}} .
\end{gathered}
$$

Here expression (21) is a special case of (19).

Case 12. McNolty [16].

$$
\begin{gathered}
f(x ; \lambda, \tau, \beta ; P, Q)=\frac{\tau^{P} \lambda^{P+Q} x^{Q-1}}{2^{Q-P}(1+2 \lambda \tau)^{P}} \cdot \exp \left(-\frac{a+b x}{1+2 \lambda \tau}\right) \\
\cdot \sum_{m=0}^{\infty} \frac{1}{m ! \Gamma(m+Q)} \cdot\left[\frac{a b x}{(1+2 \lambda \tau)^{2}}\right]^{m} \cdot{ }_{1} F_{1}\left[Q-P ; m+Q ;-\frac{\lambda x}{2(1+2 \lambda \tau)}\right], \\
x \geqq 0, \\
a=\beta^{2} / 4 \tau, b=\lambda^{2} \tau ; \lambda, \tau, \beta \geqq 0 ; P, Q>0 .
\end{gathered}
$$

$$
Q(t)=\frac{\tau^{P} \lambda^{P+Q}}{(\lambda-2 i t)^{0-P}\left[\lambda^{2} \tau-i t(1+2 \lambda \tau)\right]^{P}} \exp \left\{\frac{\beta^{2} i t}{4 \tau\left[\lambda^{2} \tau-i t(1+2 \lambda \tau)\right]}\right\} .
$$

Case 13.

$f(x)=\frac{\beta^{c} \cdot \exp \left(-\gamma^{2} / 4 \beta\right) x^{(a+b) / 2-1} \cdot(-1)}{\Gamma(c) 2^{(a+b) / 2}(\beta+1)^{c}}$

(25a)

$$
\cdot \sum_{n=0}^{\infty} \frac{\Gamma(n+c) x^{n}{ }_{1} F_{1}\left[n+c ; c ; \frac{\gamma^{2}}{4(\beta+1)}\right]}{(\beta+1)^{n} n ! \Gamma(a+n) 2^{n}} W_{(a-b) / 2,(a+b+2 n-1) / 2}(2 x) .
$$

$x>0$,

$$
a, b, c>0 ; \gamma, \beta \geqq 0 ; a+b>1,
$$

$(25 b)=(-1) \cdot[$ expression $(25 a)$ with $x$ replaced by $(-x)$ and $\Gamma(a+n)$ replaced by $\Gamma(b+n)], \quad x<0$,

$$
\varphi(t)=\frac{\beta^{c}\left(1+t^{2}\right)^{c}}{(1-i t)^{a}(1+i t)^{b}\left[\beta+(1+\beta) t^{2}\right]^{c}} \cdot \exp \left\{-\frac{\gamma^{2} t^{2}}{4 \beta\left[\beta+(1+\beta) t^{2}\right]}\right\} .
$$

Case 14.

$$
\begin{gathered}
f(x)=\frac{\pi^{1 / 2}\left(b^{2}-a^{2}\right)^{\nu+1 / 2} x^{(c+d) / 2-1}}{(2 b+1)^{2 \nu+1} \Gamma(\nu+1) \Gamma\left(\nu+\frac{1}{2}\right) 2^{(c+d) / 2-1}} \sum_{n=0}^{\infty} \frac{(-1) x^{n} \Gamma(n+2 \nu+1)}{n ! \Gamma(c+n) 2^{n}(2 b+1)^{n}} \\
\cdot{ }_{2} F_{1}\left[\frac{n}{2}+\nu+\frac{1}{2}, \frac{n}{2}+\nu+1 ; \nu+1 ; \frac{4 a^{2}}{(2 b+1)^{2}}\right] \\
\cdot W_{(c-d) / 2,(c+d+2 n-1) / 2}(2 x), \quad x>0,
\end{gathered}
$$

$$
c, d>0 ; b>a>0 ; \nu>-\frac{1}{2},
$$

$$
=(-1) \cdot[\text { expression }(27 a) \text { with } x \text { replaced by }(-x)
$$
and $\Gamma(c+n)$ replaced by $\Gamma(b+n)], \quad x<0$, 
(28) $\varphi(t)=\frac{2^{2 v+1}\left(b^{2}-a^{2}\right)^{\nu+1 / 2}\left(1+t^{2}\right)^{2 \nu+1}}{\left\{4\left(b^{2}-a^{2}\right)+4 t^{2}\left[b(1+2 b)-2 a^{2}\right]+t^{4}\left[(1+2 b)^{2}-4 a^{2}\right]\right\}^{\nu+1 / 2} \cdot(1-i t)^{c}(1+i t)^{d}}$.

Case 15.

$$
\begin{gathered}
f(x)=\frac{a^{c+\gamma-\beta}(a-b)^{\beta} \lambda^{c+d}(2 \theta)^{d} \cdot \exp \left(-a x-\frac{\phi^{2}}{4 \lambda}\right)}{\Gamma(\gamma) \Gamma(c) \Gamma(d)(1+2 \lambda \theta)^{d}(2+a \lambda)^{c}} x^{\gamma-1} \\
\cdot \sum_{m=0}^{\infty} \sum_{n=0}^{\infty} \sum_{r=0}^{\infty} \frac{(\beta)_{m}(c+n)_{r}(2 a)^{n}(a \lambda)^{r} b^{m} \Gamma(c+n) \Gamma(d+r)}{(\gamma)_{m+n}(c)_{r} m ! n ! r !(2+a \lambda)^{n+r}(1+2 \lambda \theta)^{r}} x^{m+n} \\
\cdot{ }_{1} F_{1}\left[d+r ; d ; \frac{\lambda \phi^{2}}{2(1+2 \lambda \theta)}\right], \quad x \geqq 0, \\
\varphi(t)=\frac{(a \lambda)^{c}(a-b)^{\beta}(a-i t)^{\beta-\gamma+c}(\theta \lambda)^{d}\left[a^{2} \lambda-i t(2+a \lambda)\right]^{d-c}}{a^{\beta-\gamma}(a-b-i t)^{\beta}\left\{a^{2} \theta \lambda^{2}-i t[1+\theta \lambda(2+a \lambda)]\right\}^{d}} \\
\cdot \exp \left\{-\frac{\phi^{2}}{4 \theta}+\frac{\lambda \phi^{2}\left[a^{2} \lambda-i t(2+a \lambda)\right]}{4 a^{2} \theta \lambda^{2}-4 i t[1+\theta \lambda(2+a \lambda)]}\right\} .
\end{gathered}
$$

Case 16.

$$
f(x)=\frac{\pi^{1 / 2} a^{2 \nu+\gamma-\beta+1}\left(d^{2}-c^{2}\right)^{\nu+1 / 2}(a-b)^{\beta} x^{\gamma-1} e^{-a x}}{2^{2 \nu}(a d+1)^{2 \nu+1} \Gamma(\gamma) \Gamma\left(\nu+\frac{1}{2}\right) \Gamma(\nu+1)} \cdot \sum_{m=0}^{\infty} \sum_{n=0}^{\infty} \frac{a^{n} \Gamma(n+2 \nu+1)}{(a d+1)^{n}(\gamma)_{m+n}}
$$

$$
\frac{(\beta)_{m} b^{m} x^{m+n}}{m ! n !}{ }_{2} F_{1}\left[\frac{n}{2}+\nu+\frac{1}{2}, \frac{n}{2}+\nu+1 ; \nu+1 ; \frac{a^{2} c^{2}}{(a d+1)^{2}}\right], \quad x \geqq 0,
$$

$$
\begin{gathered}
a>b \geqq 0 ; \gamma>0 ; \beta \geqq 0 ; d>c>0 ; \nu>-\frac{1}{2}, \\
\varphi(t)=\frac{(a-b)^{\beta}(a-i t)^{\beta+2 \nu-\gamma+1} \cdot a^{2 \nu-\beta+\gamma+1}\left(d^{2}-c^{2}\right)^{\nu+1 / 2}}{(a-i t-b)^{\beta}\left\{\left[a^{2} d-i t(1+a d)\right]^{2}-a^{2} c^{2}(a-i t)^{2}\right\}^{\nu+1 / 2}} .
\end{gathered}
$$

Case 17.

$$
\begin{aligned}
& f(x)=\frac{a^{\lambda+b \mu+1} \lambda^{b} \cdot \exp \left(-a x-\frac{\tau^{2}}{4 \lambda}\right) \cdot x^{\lambda}}{\Gamma(b)\left(2+\lambda a^{\mu}\right)^{b}} \\
& \text { (33) } \cdot \sum_{n=0}^{\infty} \frac{2^{n} a^{n \mu} \Gamma(b+n) x^{n \mu}}{n !\left(2+\lambda a^{\mu}\right)^{n} \Gamma(1+\lambda+n \mu)} \cdot{ }_{1} F_{1}\left[b+n ; b ; \frac{a^{\mu} \tau^{2}}{4\left(2+\lambda a^{\mu}\right)}\right], \quad x \geqq 0,
\end{aligned}
$$

$$
\begin{gathered}
a, b, \lambda>0 ; \mu \geqq-1 ; \tau \geqq 0, \\
\varphi(t)=\frac{\left(\lambda a^{\mu}\right)^{b}(a-i t)^{b \mu} \cdot \exp \left(-\frac{\tau^{2}}{4 \lambda}\right) \cdot a^{\lambda+1}}{(a-i t)^{\lambda+1}\left[(a-i t)^{\mu}\left(2+\lambda a^{\mu}\right)-2 a^{\mu}\right]^{b}} \\
\cdot \exp \left[\frac{a^{\mu} \tau^{2}(a-i t)^{\mu}}{4(a-i t)^{\mu}\left(2+\lambda a^{\mu}\right)-8 a^{\mu}}\right] .
\end{gathered}
$$


Case 18.

$$
\begin{aligned}
& f(x)= \frac{\pi^{1 / 2}\left(b^{2}-a^{2}\right)^{\nu+1 / 2} c^{2 \mu \nu+\mu+\lambda+1} x^{\lambda} e^{-c x}}{2^{2 \nu}\left(b c^{\mu}+1\right)^{2 \nu+1} \Gamma(\nu+1) \Gamma\left(\nu+\frac{1}{2}\right)} \sum_{n=0}^{\infty} \frac{\Gamma(2 \nu+n+1) c^{n \mu} x^{n \mu}}{\left(b c^{\mu}+1\right)^{n} n ! \Gamma(1+\lambda+n \mu)} \\
& \cdot{ }_{2} F_{1}\left[\nu+\frac{n}{2}+\frac{1}{2}, \nu+\frac{n}{2}+1 ; \nu+1 ; \frac{a^{2} c^{2 \mu}}{\left(b c^{\mu}+1\right)^{2}}\right], \quad x \geqq 0, \\
& b>a>0 ; c, \lambda>0 ; \nu>-\frac{1}{2} ; \mu \geqq-1, \\
& \varphi(t)=\frac{\left(b^{2}-a^{2}\right)^{\nu+1 / 2} \cdot c^{2 \mu \nu+\mu}(c-i t)^{2 \mu \nu+\mu} \cdot c^{\lambda+1}}{(c-i t)^{\lambda+1}\left\{\left[(c-i t)^{\mu}\left(1+b c^{\mu}\right)-c^{\mu}\right]^{2}-a^{2} c^{2 \mu}(c-i t)^{2 \mu}\right\}^{\nu+1 / 2}} .
\end{aligned}
$$

Case 19. McNolty [23], [24].

$$
\begin{gathered}
f(x)=x^{2} e^{-\tau x^{2}} / 2 K\left(a+b x^{2}+c x^{4}\right), \quad-\infty<x<\infty, \\
a, b, c>0 ; \tau \geqq 0,
\end{gathered}
$$

where

$$
K=\frac{\pi^{1 / 2}}{4 c\left(r_{2}-r_{1}\right)}\left[r_{1}^{1 / 2} e^{r_{1} \tau} \Gamma\left(-\frac{1}{2}, r_{1} \tau\right)-{r_{2}}^{1 / 2} e^{r_{2} \tau} \Gamma\left(-\frac{1}{2}, r_{2} \tau\right)\right]
$$

and $\Gamma(a, x)$ is the incomplete gamma function, $\Gamma(a, x)=\Gamma(a)-\gamma(a, x)$.

$$
\varphi(t)=\frac{\pi^{1 / 2}}{8 K c\left(r_{1}-r_{2}\right)} \int_{\tau}^{\infty} \frac{2 u-t^{2}}{u^{5 / 2}} \cdot e^{-t^{2 / 4 u}} \cdot\left[e^{r_{2}(\tau-u)}-e^{r_{1}(\tau-u)}\right] d u,
$$

where $r_{1}, r_{2}$ are the two roots (which may be complex) given by

$$
r_{1}, r_{2}=\frac{ \pm b \pm\left(b^{2}-4 a c\right)^{1 / 2}}{2 c}, \quad b^{2} \neq 4 a c
$$

Case 19 was developed jointly by the author and Dr. Eldon Hansen of the Lockheed Palo Alto Research Laboratory. Expression (37) is an important function occurring in the design of optimum infrared signal processors. Other functions listed above occur in problems related to: fluctuating radar cross section, fading radio signals, weapons coverage and distributions of sums of random variables.

In expression (38), the restriction is $r_{1} \neq r_{2}$, i.e., $b^{2}-4 a c \neq 0$. If we require that $b^{2}-4 a c>0$ (which is the usual case in applications), then (38) becomes

$$
\begin{aligned}
\varphi(t)=\frac{\pi}{2 K c\left(N^{2}-Q^{2}\right)}\left\{N e^{-N t+\tau N^{2}} \cdot\left[\Phi\left(\frac{t}{(4 \tau)^{1 / 2}}-N \tau^{1 / 2}\right)+1\right]\right. \\
-N e^{N t+\tau N^{2}} \cdot\left[\Phi\left(\frac{t}{(4 \tau)^{1 / 2}}+N \tau^{1 / 2}\right)+1\right] \\
-Q e^{-0 t+\tau Q^{2}} \cdot\left[\Phi \left(\frac{t}{\left.\left.(4 \tau)^{1 / 2}-Q \tau^{1 / 2}\right)+1\right]+Q e^{Q t+\tau \theta^{2}}}\right.\right. \\
\left.\cdot\left[\Phi\left(\frac{t}{(4 \tau)^{1 / 2}}+Q \tau^{1 / 2}\right)+1\right]\right\},
\end{aligned}
$$

where, in (38a), $N=(b / 2 c-M)^{1 / 2}, Q=(b / 2 c+M)^{1 / 2}, N^{2} Q^{2}=a / c, N^{2}+Q^{2}=$ $b / c, M=\left(b^{2}-4 a c\right) / 4 c^{2}, K$ is the normalizing factor when $t=0$ and $\Phi(x)=\operatorname{erf}(x)$. 
The restriction $b^{2}-4 a c>0$ (with $a, b, c>0$ ) implies that the four poles of (37) must lie (in two conjugate pairs) on the imaginary axis of the $z=x+i y$ plane rather than falling in arbitrary locations as is the case when the constraint is simply $r_{1} \neq r_{2}$.

Continuous Distributions. References [27] and [28] by Norman L. Johnson and Samuel Kotz provide an excellent treatment of the subject of continuous distributions. The two volumes contain an extremely large collection of distributions together with lucid discussions of their properties and applications.

Mixture Representations. As an alternative to expressing the preceding functions in terms of special functions, one can write them as mixture representations (Luke [13] and McNolty [20]), i.e., infinite series of simpler density functions weighted by discrete probability functions. Several examples are given below. In what follows, $P(n ; a)$ is the Poisson distribution on $n$ with parameter $a, N B(m ; a, b)$ is the negative binomial distribution on $m$ with parameters $a$ and $b$ and $G(u ; a, b)$ is the continuous gamma distribution for $u$ with parameters $a$ and $b$.

A. Expression (1) can be written as

$$
f(x ; \lambda, \gamma, Q)=\sum_{m=0}^{\infty} P\left(m ; \frac{\gamma^{2}}{4 \lambda}\right) \cdot G\left(x ; \frac{\lambda}{2}, Q+m\right), \quad x \geqq 0 .
$$

B. Expression (5) becomes

$$
f(x ; a, b ; \lambda, \mu)=\sum_{m=0}^{\infty} P\left(m ; \frac{b}{a^{\mu}}\right) \cdot G(x ; a, m \mu+\lambda+1), \quad x \geqq 0 .
$$

C. Expression (11) is

$$
f(x ; a, b ; \nu, \mu)=\sum_{n=0}^{\infty} N B\left(n ; \nu, \frac{a}{b}\right) \cdot G(x ; b, \mu+\nu+n), \quad x \geqq 0 .
$$

D. Expression (17) is given by

$$
\begin{aligned}
f(x ; a, b, c ; \beta, \gamma)=\sum_{m=0}^{\infty} \sum_{n=0}^{\infty} P\left(n ; \frac{c}{a}\right) \cdot N B\left(m ; \beta, 1-\frac{b}{a}\right) \\
\cdot G(x ; a, \gamma+m+n), \quad x \geqq 0 .
\end{aligned}
$$

E. Expression (19) is

$$
\begin{aligned}
f(x ; a, b, c, d ; \beta, \gamma, \lambda, \tau) & =\sum_{m=0}^{\infty} \sum_{n=0}^{\infty} \sum_{r=0}^{\infty} N B\left(m ; b, 1-\frac{\lambda}{a}\right) N B\left(n ; c, 1-\frac{\tau}{a}\right) \\
\cdot & N B\left(r ; d, 1-\frac{\beta}{a}\right) G(x ; a, m+n+r+\gamma), \quad x \geqq 0 .
\end{aligned}
$$

F. Expression (21) is

$$
\begin{aligned}
& f(x ; a, b, c ; \gamma ; \lambda, \tau)=\sum_{m=0} \sum_{n=0} N B\left(m ; b, 1-\frac{\lambda}{a}\right) \cdot N B\left(n ; c, 1-\frac{\tau}{a}\right) \\
& \cdot G(x ; a, m+n+\gamma), \quad x \geqq 0 .
\end{aligned}
$$


Other functions from Cases 1 through 19 may be written in a similar manner and, thus, a two-fold description of these densities in terms of both special functions and mixture representations can provide additional insight into their mathematical structure. Also, in performing various manipulations with the density functions one has a choice of using the special-function form or the mixture representation. One or the other might be easier to work with depending upon the problem. The extensive discussion in Chapter 9 of Luke [13] provides more generalized results than the simple expansions (1), (5), (11), (17), (19) and (21) above.

Sums of Random Variables. Many of the functions presented in Cases 1 through 19 reproduce themselves by addition of independent variables. The reproductive property holds true provided that certain of the parameters remain fixed for each variate in the sum, while other parameters need not remain the same. The fixed parameters ordinarily correspond to the location of the poles, zeros or branch points of the characteristic functions; while the "free" parameters are those which determine the order of the poles or zeros or the number of branches of the function. The characteristic functions are infinitely divisible with respect to the free parameters. Clearly, for instance, the distribution (15) is not reproduced under the addition of independent random variables.

Although the examples which follow are purely illustrative and the notation imperfect, the results indicate the application of the preceding density functions to addition problems.

The random variable $X_{\diamond}(u ; \alpha, \lambda)$ will be distributed according to the gamma distribution

$$
\begin{gathered}
g(u ; \alpha, \lambda)=\alpha^{\lambda} u^{\lambda-1} e^{-\alpha u} / \Gamma(\lambda), \quad u \geqq 0, \\
\alpha, \lambda>0,
\end{gathered}
$$

while the random variables $X_{B}(u ; \lambda, \gamma, Q)$ and $X_{h}(u ; a, b ; \nu, \mu)$ are distributed according to expressions (1) and (11), respectively. Similarly,

$$
X_{K}(v ; a, \nu), X_{\Phi_{3}}(u ; a, b ; c ; \beta, \gamma), X_{w}(u ; a, b ; c) \quad \text { and } \quad X_{\Phi_{2}}(u ; a, b, c ; \gamma ; \lambda, \tau)
$$

denote random variables distributed according to (3), (17), (7) and (21), respectively. Then the following relationships hold true:

(a) For $U=X+Y$,

$$
X_{h}(u ; a, b ; \nu, \mu)=X_{o}(x ; a, \nu)+X_{o}(y ; b, \mu)
$$

where $a, b \geqq 0$ and now $\nu>0$ and $\mu>0$, rather than $\mu+\nu>0$.

(b) For $V=X-Y$,

$$
X_{K}(v ; a, \nu)=X_{0}\left(x ; a, \nu+\frac{1}{2}\right)-X_{0}\left(y ; a, \nu+\frac{1}{2}\right)
$$

where $a>0$ and $\nu=-\frac{1}{2}$.

(c) For $U=X+Y$,

$$
X_{\Phi_{3}}(u ; a, b ; c ; \beta, \gamma)=X_{B}\left(x ; 2 a, 2(2 c)^{1 / 2}, \gamma_{2}\right)+X_{h}\left(y ; a-b, a ; \beta, \gamma_{1}-\beta\right)
$$

where $\gamma=\gamma_{1}+\gamma_{2} ; a>b \geqq 0 ; c, \beta \geqq 0 ; \gamma, \gamma_{1}, \gamma_{2}>0$ and, if further, $\gamma_{1}-\beta>0$ then for $U=X+Z+W$ we write 
$X_{\Phi_{3}}(u ; a, b ; c ; \beta, \gamma)$

$$
=X_{B}\left(x ; 2 a, 2(2 c)^{1 / 2}, \gamma_{2}\right)+X_{o}(z ; a-b, \beta)+X_{o}\left(w ; a, \gamma_{1}-\beta\right) .
$$

(d) For $U=X-Y$,

$$
X_{w}(u ; a, b ; c)=X_{B}\left(x ; 2,2^{1 / 2} c, a\right)-X_{B}\left(y ; 2,2^{1 / 2} c, b\right)
$$

where, of course, the appearance of a Bessel variate always permits a further resolution into sums of Bessel variates and/or gamma distributed variates.

(e) For $U=X+Y$,

$$
X_{\Phi_{2}}(u ; a, b, c ; \gamma ; \lambda, \tau)=X_{h}(x ; a-\lambda, a ; b, \gamma-b-c)+X_{0}(y ; a-\tau, c)
$$

when $c>0$ and $\gamma-c>0$ where $a>\lambda ; a>\tau ; a, b>0 ; \lambda, \tau \geqq 0$.

(f) For $U=X+Y$,

$$
X_{\Phi_{2}}(u ; a ; b, c ; \gamma ; \lambda, \tau)=X_{h}(x ; a-\tau, a ; c, \gamma-b-c)+X_{o}(y ; a-\lambda, b)
$$

when $b>0$ and $\gamma-b>0$ where $a>\lambda, a>\tau ; a, c>0 ; \lambda, \tau \geqq 0$.

(g) For $U=X+Y$,

$$
X_{\Phi_{2}}(u ; a ; b, c ; \gamma ; \lambda, \tau)=X_{h}(x ; a-\lambda, a-\tau ; b, c)+X_{o}(y ; a, \gamma-b-c)
$$

when $b+c>0, \gamma-b-c>0$ where $a>\lambda ; a>\tau$;

$$
a>0 ; \lambda, \tau \geqq 0 ; \text { and }\left\{\begin{array}{l}
b>0 \text { if } a-\tau>a-\lambda, \\
c>0 \text { if } a-\lambda>a-\tau .
\end{array}\right.
$$

(h) For $U=X+Y+Z$,

$X_{\Phi_{2}}(u ; a ; b, c ; \gamma ; \lambda, \tau)$

$$
=X_{o}(x ; a-\lambda, b)+X_{o}(y ; a-\tau, c)+X_{0}(z ; a, \gamma-b-c)
$$

when $b>0, c>0$ and $\gamma-b-c>0$ where $\lambda, \tau \geqq 0 ; a>\lambda, a>\tau, a>0$.

(i) Denote the random variable distributed according to (15) by $X_{I}(x ; a, b ; \nu)$ then for $U=X+Y$,

$$
X_{I}(u ; a, b ; \nu)=X_{h}\left(x ; b-a, b ; \nu+\frac{1}{2},-1\right)+X_{o}\left(y ; b+a, \nu+\frac{1}{2}\right)
$$

where now $\nu>\frac{1}{2} ; b>a>0$.

(j) Denote the random variable distributed according to (25) by $X$, then, for $X=X_{1}-X_{2}+X_{3}-X_{4}$,

$$
\begin{aligned}
X= & X_{B}\left(x_{1} ; 2\left(\frac{\beta}{1+\beta}\right)^{1 / 2}, \frac{\gamma^{2}}{(2 \beta)^{1 / 2}}(1+\beta)^{3 / 2}, c\right) \\
& -X_{B}\left(x_{2} ; 2\left(\frac{\beta}{1+\beta}\right)^{1 / 2}, \frac{\gamma^{2}}{(2 \beta)^{1 / 2}(1+\beta)^{3 / 2}}, c\right) \\
& +X_{h}\left(x_{3} ; 1,\left(\frac{\beta}{1+\beta}\right)^{1 / 2} ; a-c, c\right)-X_{h}\left(x_{4} ; 1,\left(\frac{\beta}{1+\beta}\right)^{1 / 2} ; b-c, c\right) .
\end{aligned}
$$

The author would like to extend his appreciation to Lill Staley, departmental secretary of the Mathematics and Operations Research Laboratory of the Lockheed 
Palo Alto Research Laboratory, for her excellent typing of this rather cumbersome text.

Lockheed Palo Alto Research Laboratory

Palo Alto, California 94305

1. B. C. Bhattacharyya, "The use of McKay's Bessel function curves for graduating frequency distributions," Sankhyā, v. 6, 1942, pp. 175-182. MR 5, 126.

2. R. C. Bose, "On the exact distribution and moment-coefficients of the $D^{2}$-statistic," Sankhyā, v. 2, 1936, pp. 143-154.

3. S. S. Bose, "On a Bessel function population," Sankhyā, v. 3, 1938, pp. 253-261.

4. A. ERdélyi, W. Magnus, F. Oberhettinger \& F. G. TRicomi, Tables of Integral Transforms, Vol. 1, McGraw-Hill, New York and London, 1954. MR 15, 868.

5. J. O. IRwIN, "On the frequency distribution of the means of samples," Biometrika, v. 19,1927 , pp. $225-239$.

6. K. V. Krishna SASTry, "On a Bessel function of the second kind and Wilks $Z$-distribution," Indian Acad. Sci. Ser. A, v. 28, 1948, pp. 532-536. MR 10, 387.

7. RadHa G. LaHa, "On some properties of the Bessel function distributions," Bull. Calcutta Math Soc., v. 46, 1954, pp. 59-72. MR 16, 152.

8. R. D. LoRD, "The use of Hankel transforms in statistics. I," Biometrika, v. 41, 1954a, pp. 44-55. MR 15, 885.

9. R. D. LoRD, "The use of the Hankel transform in statistics. II," Biometrika, v. 41, 1954b, pp. 344-350. MR 16, 382.

10. R. D. LoRD, "The distribution of distance in a hypersphere," Ann. Math. Statist., v. 25, 1954c, pp. 794-798. MR 16, 377.

11. YUdell L. LuKE, Integrals of Bessel Functions, McGraw-Hill, New York and London, 1962. MR 25 \#5198.

12. Yudell L. LuKe, The Spectral Functions and Their Approximations, Vol. 1, Math. in Sci. and Engineering, vol. 53, Academic Press, New York, 1969. MR 39 \#3039.

13. Yudell L. LukE, The Special Functions and Their Approximations. Vol. 2, Math. in Sci. and Engineering, vol. 53, Academic Press, New York, 1969. MR 40 \#2909.

14. A. T. MCKaY, "A Bessel function distribution," Biometrika, v. 24, 1931, pp. 39-44.

15. FRANK MCNOLTY, "A contour-integral derivation of the non-central chi-square distribution," Ann. Math. Statist., v. 33, 1962, pp. 796-800. MR 25 \#651.

16. FRANK MCNolTy, "Applications of Bessel function distributions," Sankhyā Ser. B, v. 29, 1967, pp. 235-248. MR 37 \#3672.

17. FranK MCNolTy, "A note on radial distributions," Operations Res., v. 16, 1968a, pp. 211-216.

18. FRANK McNolty, "Expected coverage for targets of nonuniform density," Operations Res., v. 16, 1968b, pp. 1027-1040.

19. FRANK MCNolty, "Quadratic form distribution associated with special functions," Sankhyā Ser. B, v. 34, 1972, pp. 21-26.

20. Frank McNolTy \& JACK TOMSKY, "Some properties of special-function, bivariate distributions," Sankhyā Ser. B, v. 34, 1972, pp. 251-264. mitted.)

21. FRANK MCNOLTY, "Random vectors with non-uniform phase distributions." (Sub-

22. Frank McNolty, "Reliability density functions when the failure rate is randomly distributed," Sankhyä Ser. A, v. 26, 1964, pp. 287-292. MR $32 \# 4719$.

23. Frank McNolty, R. Clow \& E. Hansen, "Some matched filter configurations for infrared systems," IEEE Trans. Aerospace and Electronic Systems, v. AES-8, 1972, pp. 428438.

24. Frank McNolty, R. Clow \& E. Hansen, "Some properties of the output of integrator in an infrared system," IEEE Trans. Aerospace and Electronic Systems, v. AES-8, 1972, pp. 552-558.

25. Karl Pearson, "Further applications in statistics of the $T_{m}(X)$ Bessel function," Biometrika, v. 24, 1932, pp. 293-350.

26. DES RAJ, "On a generalized Bessel function population," Ganita, v. 3, 1953, pp. 111115. MR 14, 775 .

27. Norman L. Johnson \& Samuel Kotz, Distributions in Statistics. Continuous Univariate Distributions. 1, Houghton Mifflin, Boston, Mass., 1970. MR 42 \#5363.

28. Norman L. Johnson \& Samuel Kotz, Distributions in Statistics. Continuous Univariate Distributions. 2, Houghton Mifflin, Boston, Mass., 1970. MR 42 \#5364. 